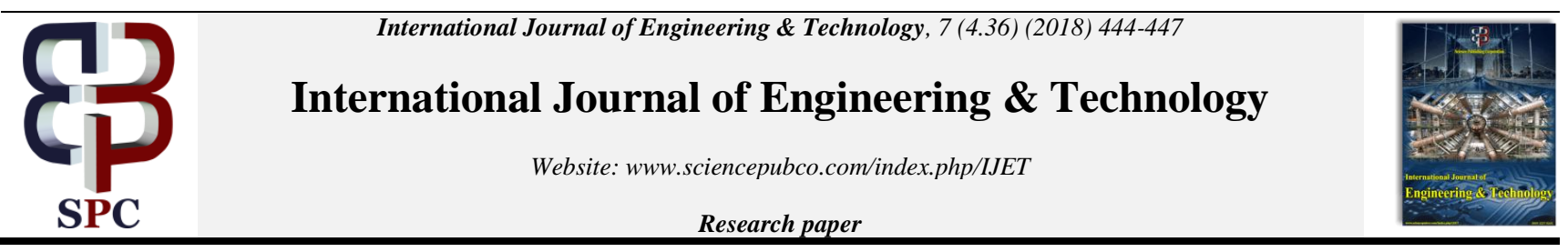

\title{
A Neural Basis for the Implementation of Deep Learning and Arti cial Intelligence
}

\author{
Alan F. Smeaton \\ Insight Centre for Data Analytics, Dublin City University, Dublin 9, Ireland \\ *Corresponding author E-mail: \{alan.smeaton\}@dcu.ie
}

\begin{abstract}
.
One of the mathematical cornerstones of modern data ana-lytics is machine learning whereby we automatically learn subtle patterns which may be hidden in training data, we associate those patterns with outcomes and we apply these patterns to new and unseen data and make predictions about as yet unseen outcomes. This form of data analytics al-lows us to bring value to the huge volumes of data that is collected from people, from the environment, from commerce, from online activities, from scienti c experiments, from many other sources. The mathematical basis for this form of machine learning has led to tools like Support Vector Machines which have shown moderate e ectiveness and good e ciency in their implementation. Recently, however, these have been usurped by the emergence of deep learning based on convolutional neural networks. In this presentation we will examine the basis for why such deep net-works are remarkably successful and accurate, their similarity to ways in which the human brain is organised, and the challenges of implementing such deep networks on conventional computer architectures.
\end{abstract}

Keywords: Deep learning, neural computing, neural networks

\section{Introduction}

\subsection{A Brief History of Computing}

The short history of the development of computing is a mostly linear progression of the same basic principle of what makes a computer. Starting with the early thoughts on stored program computers by members of the team developing the ENIAC computer at the end of WW II and then leading on to the work of Alan Turing, this basic principle is about a device that stores a sequence of instructions to be executed in sequence, one after the other. We have seen major technological developments like the transistor in 1947, the integrated circuit in 1959, di erent forms of magnetic memory for storing instructions and data, and on the software side we have seen mainframes evolve into personal computers and now even wearable technology. The common characteristic across all these developments is the stored program model for computers proposed by John von Neumann in 1952. Here, a central processing unit retrieves an instruction from memory, decodes it, executes it and then moves on to fetch and then execute the next instruction unless directed to fetch the next instruction from somewhere else, as shown in Figure 1. This von Neumann architecture has not only carried computing since the advent of digital computation 60 or 70 years ago, but it is the basis for almost all kinds of computing that we perform today.

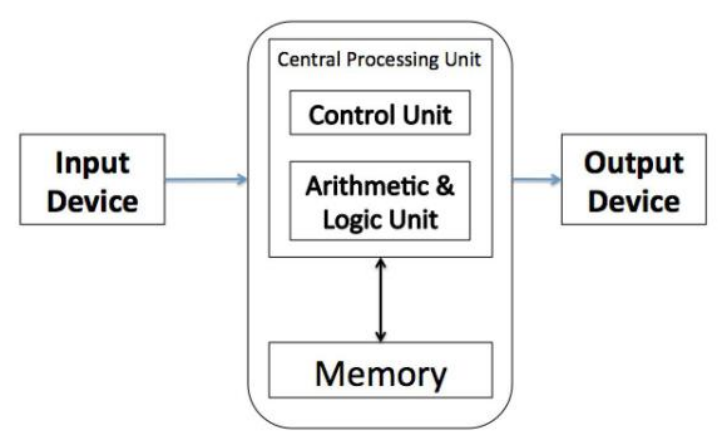

Fig. 1: The traditional von Neumann architecture for computers

\section{Arti cial Intelligence}

\subsection{Arti cial Intelligence Emerges}

Even since those early days, the question has been asked of whether computers could perform an arti cial form of intelligence or execute tasks at the human level of performance. To do this we need to mimic the human brain, which although making up on $2 \%$ of our body weight, is made up of more than 80 billion neurons connected by trillions of connections or synapses. The brain is responsible for our executive functions like breathing, digestion, heart pumping, as well as even more complex tasks like planning, reasoning, and abstract thought. It achieves this using an architecture of a huge number (+80B) of simple, connected processors.

This architecture is good for solving complex problems like vision, and learn-ing. The unit components of this architecture are neurons or simple perceptrons. Each neuron is on average 
connected to about 10,000 other neurons and neurons communicate by sending signals across these synapses. They operate by receiv-ing a number of input signals from the multiple input connections or channels that they each have, aggregating the input signals in some way, called an acti-vation function, and then based on the values of the input signals, they generate an output signal which is passed on to another neuron to which it is connected. While each neuron is very simple, it is the huge number of them, and their con-nections, that allow the overall system to address complex problem-solving, the kind that we call arti cial intelligence.

So how do we implement arti cial intelligence tasks, emulating the complex-ity of the human brain, on the von Neumann architecture which is so prevalent today? Well clearly the von Neumann architecture with its sequential process-ing of instructions and in-built lack of parallelism, is clearly not a good $\mathrm{t}$ for implementing neural computing. In the late 1980s we saw the rst attempts at implementing a neural architecture directly in hardware through the devel-opment of the Connection Machine by the Thinking Machines Corp. While a faithful replication of neural information processing, the problem with the Con-nection Machine was that it only had some thousands of nodes with connections which was not enough to simulate the kind of intelligence at the level we seek for human problem-solving, so it was used for simple, massive parallelism appli-cations like searching.

\section{2}

The Arti cial Intelligence Winter

Around the time of the Connection Machine we were entering into the \AI Winter" with in ated expectations around AI leading to disappointment in the actual realization and a very deep Itrough of disillusionment" as found in the classic Gartner curve. Research into neural networks | computational imple-mentations of the brains structure $\mid$ continued but at a slow rate of progress and forming a bit of an AI backwater. Meanwhile hand-crafted, rules-driven arti cial intelligence research continued through the 1970s, 80s, 90s, and even 00s, with progress in AI applications in areas like speech, machine translation, computer visions and expert systems, for a while anyway.

The area of neural networks, while not hugely popular or attracting much research interest or funding, did continue to develop during this period and developed new con gurations of neural networks like multi-layer perceptrons, convolutional neural networks [5], spiking neural networks [6], and more.

The simple con guration of a neuron is shown in Figure 2 where on the left side we show a simple neuron with 2 input connections, though there can be many more. The neuron accepts its two input signals, performs some operation on the signal values to generate an output signal which is sends. Neural networks are generally con gured to form feed forward networks, operating on a set of inputs and generating one or more outputs. A simple feed-forward network is shown on the right in Figure 2 with 2 inputs,

Neural network architectures are much more complex that that shown in Figure 2 because they are so numerous. They usually have a number of hidden layers, an input layer, and an output layer. Adjacent layers are usually fully connected where every neuron in one layer is connected to every neuron in an-other layer. Layers can also have loops back to earlier layers, called recurrent neural networks, increasing both their complexity and the complexity of their problem-solving capabilities [13].

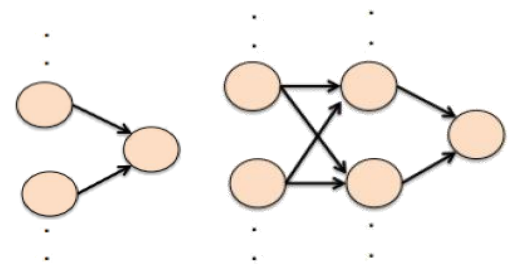

Fig. 2: Architecture components in neural networks: simple neuron (left) and feed forward network (right)

\subsection{The Growth of Machine Learning}

Meanwhile, as neural network architectures were developing, in other parts of the arti cial intelligence area, machine learning developed as an AI tool and its development hinged on a background of mathematics and statistics rather than any idea of emulating human neural processing. Machine learning evolved slowly over these decades. It was nourished by the increasing availability of huge volumes of data from sources like internet searching, social media, online transactions, and others. One application area which pushed this development was computer vision and in this eld we were able to use machine learning to train classi ers to identify objects and concepts appearing in images. To do this we used techniques like decision tree learning, random forests, genetic programming and most especially, support vector machines. The basic approach here was to use a large number of positive and negative examples, to extract lowlevel image features like shape, colour and texture, and let the computer gure out (or learn) how to classify new and unseen examples as to the presence or absence of these objects and concepts.

Up to the early and mid-point of the current decade, this worked $\mathrm{OK}$ and there was slow and incremental progress year-on-year but the techniques were not mature or accurate enough to be used in commercial or real applications; we had more work to do. At the same time, we also saw machine learning being used elsewhere in recommender systems for example, and this increased the popularity and visibility of machine learning as an AI technique. This standard or Iclassic" machine learning has several advantages including the fact that a decision or recommendation can be explained by examining the features or characteristics of each case, or recommendation. We can also rate the relative importance of each of the features or axes which gives us unique insights into the problem we are trying to solve. However, a downside here is that we have to do lots of feature engineering to de ne the axes of the problem space and there are no theoretical underpinnings to this and so it is a form of a black art.

\section{Convolutional Neural Networks}

\subsection{What is a Convolutional Neural Network?}

Convolutional Neural Networks (CNNs) are a particularly complex architecture which have been around for more than 20 years but rst really got noticed in [9]. Also known as a deep convolutional networks, they were developed to address image processing applications because their design allows an entire image to be fed into the network, so in theory there are a huge number of inputs to the network. The typical application for which CNNs were developed were to injest an entire image and to classify it as containing objects or concepts like lcat" or \dog" or lairplane" or loutdoor" or anything else that can be recognised visually.

However, rather than have an input node for each pixel in an image, which would lose whatever locality and proximity gives rise to us recognising cats, and dogs and airplanes and whatever else, CNNs create an input layer consisting of a window, a kind of sub-image, which slides across and scans the full image. This can be seen in Figure 3 where the input image is 3232 pixels and a sliding window of 553 corresponding to 55 pixels and 3 colours, and this window slides across the image, one pixel at a time, generating local context information to help recognise objects and concepts. This input is fed through convolutional layers where each node is not connected to every other one but to its local neighbourhood, and this structure is repeated a number of times. This is descried in more detail in [9]. 


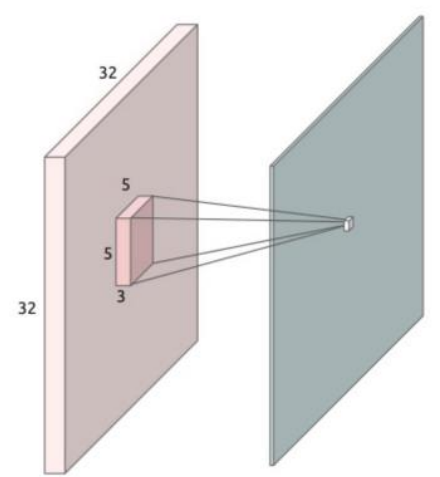

Fig. 3: Convolutional Neural Network showing sliding window in input layer

The idea of having such convolutions in a multi-layer network really gained notoriety in 2012, when Krizhevsky and his colleagues submitted a run to the ImageNet large scale visual recognition challenge based on an implementation of a convolutional neural network, and the performance of the submission was a huge improvement on the then state-of-the-art [8]. The technique was based on an architecture consisting of multiple layers of neurons, implemented using graphics processing units (GPUs) which allowed a huge increase in the number of nodes, and connections in the neural network. A schematic for such a networ is shown in Figure 4.

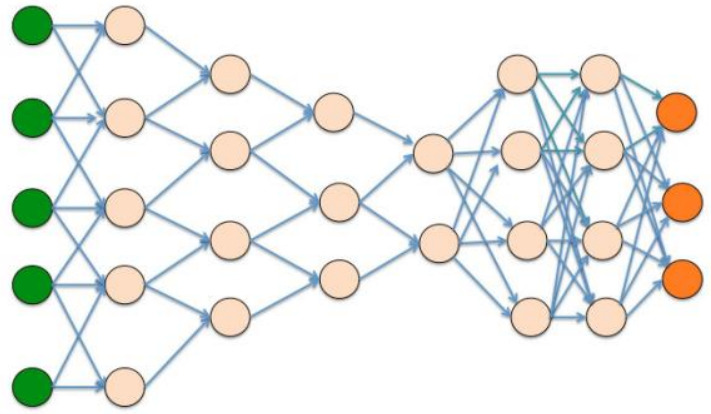

Fig. 4: Architecture of a Convolutional Neural Network

Convolutional Neural Networks are end-to-end solutions in which both the feature extraction covering features like colours, shapes and textures, and the classi er training are performed at once. These Ideep features" turn out to be signi cantly more e cient than the classical ones which heretofore had been manually engineered, even when used with classical machine learning for classi er training.

Once a model for learning a concept is built, it can be packaged and easily run in a hosted environment and this has led to a huge uptake of this ldeep learning" for computer vision problems. Google, Facebook and other major soft-ware companies now use deep learning as part of their commodity o erings for image tagging, video captioning, and other computer vision problems. To illustrate, many of the participants in TRECVid for concept detection, video captioning and other vision processing (as opposed to video search), now use CNN approaches [1].

\subsection{Applications for $\mathrm{CNNs}$}

The use of deep learning via CNNs is now almost everywhere and being used for things like speech processing [5], circuit design [14], language processing [7], machine translation [2, 11] and others. These are nicely summarised by Le Cun et al. in [11]. Other applications in other areas include automative speci cally self-driving cars, in banking it includes evaluating credit applications and evalu-ation of mortgage applications, layout of integrated circuits, predicting currency prices, predicting faults during a manufacturing process, analysis of EEG, ECG and
fMRI signals, route and movement planning in robotics, vehicle and route scheduling in transport, and many more. Deng [4] shows this even broader range of applications for CNNs and deep learning which are showing some success, but we don't know whether this is only because of parallelism and whether they actually need a neural architecture which uses layers, or other even more complex structures like those described in [3].

\subsection{Implementing Neural Networks}

Implementing convolutional neural networks and similar deep learning archi-tectures is de nitely not suitable for a von Neumann architecture so for now, many implementations use graphical processing units (GPUs) which o er mas-sive parallelism at a very cheap rate designed, as they are, for supporting graphics processing for applications like gaming.

For more longer-term implementations, we need to design new hardware chips to implement this new architecture, or rather this old architecture which has re-cently become popular. Intel are developing deep learning chips code-named Lake Crest and Knights Crest, while Samsung are targeting chips for handset devices which allow deep learning on devices. Other companies like Movidius, now part of Intel, are specialising in computer vision using deep learning on silicon. Recognising the emergent importance of deep learning and the computa-tional expense this would require for applications like speech and vision, Google designed and built a new chip from scratch called the Tensor Processing Unit which implements deep learning on a neural architecture which is 30 times faster than on a von Neumann architecture.

\subsection{Future for Neural Networks}

Neural networks are a computer architecture which is proving to be very good at some kinds of human cognitive processing . . . things like computer vision, speech, or other applications where we need to generalise from very large amounts of data. The quality of performance for some of these tasks is as good as, or better, than human processing. As such, they are attracting a lot of interest not just from the research community but also from business and commerce. As we saw above, faster implementations of neural networks are a particular focus at present, as is the move to support deel learning on mobile platforms with minimal energy consumption for extended battery life.

However besides implementation there are other areas where Neural Networks are a focus of much interest. One of these is the ability for a network, or indeed any machine learning implementation, to explain its decisions. Machine learning is used for two kinds of application . . . those where we might need to explain a decision made by an algorithm and those where no explanation might ever be needed, or indeed even be possible. Examples of the former might be credit ratings, mortgage or loan applications or grading student projects while examples of the latter might be routing in car navigation, or object recognition in computer vision. When deep learning is used, as it is increasingly, for machine learning applications like credit ratings or grading student projects, it is unable to provide an explanation as to why a particular loan application was refused or granted or why a medical X-ray had a certain medical diagnosis. Sometimes we don't care about the lwhy" associated with a decision, sometimes we do, but deep learning and neural networks by their very nature, are not designed to be able to explain why, just to decide on the output as rapidly and as accurately as possible and when explanaining does matter, neural networks can't help.

Another topic of importance in the future of neural networks is their ability to address continuous streams of input data, just like the human brain does. In present con gurations, a set of inputs are passed into the input layer of a neural network, these work their 
way through the network until they generate an out-put. When that is completed, a new set of inputs are sent to the input layer, and so on. In real work uses of neurel networks, i.e. the brain, the input layer is fed with a continuous stream of data through our senses \{ sight, hearing, etc. One Neural network architecture which addresses this are pulsed neural networks, rst introduced in [10]. This was then re ned into spiking neural networks in, for example, [6] and there is a good review of pulse-coupled neural networks in

[12].

The pulse-coupled neural network is a Isingle layer, two-dimensional, later-ally connected network of integrate-and- re neurons, with a 1:1 correspondence between the image pixels and network neurons" [12]. This means it is complex, more complex than a $\mathrm{CNN}$, and it is dynamic in that it adapts to its input, and it is able to take input data on a continuous basis.

\section{Conclusions}

Since the early days of computing we are used to having people writing algo-rithms, encoding these as programs which we store and run on von Neumann type architectures. Now, with the emergence of data-driven, AI-based technolo-gies we have data which we use to build and train networks which emulate neural networks, and we develop models to solve problems like classi cation and recognition, which we store and run. This represents the greatest single shift in computing, notwithstanding the major technological contributions of things like the transistor, integrated circuit or magnetic memory storage. Understanding this shift, maximizing its impact and using it for the most appropriate kinds of application are things we hope we can do correctly.

\section{Acknowledgements}

This work is supported by Science Foundation Ireland under grant number $12 / \mathrm{RC} / 2289$.

\section{References}

[1] G. Awad, J. Fiscus, D. Joy, M. Michel, A. F. Smeaton, W. Kraaij, G. Quenot, M. Eskevich, R. Aly, R. Ordelman, et al. Trecvid 2016: Evaluating video search, video event detection, localization, and hyperlinking. In Proceedings of TRECVID, volume 2016, 2016.

[2] K. Cho, B. Van Merri•enboer, D. Bahdanau, and Y. Bengio. On the proper-ties of neural machine translation: Encoder-decoder approaches. arXiv preprint arXiv:1409.1259, 2014.

[3] H. B. Demuth, M. H. Beale, O. De Jess, and M. T. Hagan. Neural Network Design. Martin Hagan, USA, 2nd edition, 2014.

[4] L. Deng. A tutorial survey of architectures, algorithms, and applications for deep learning. APSIPA Transactions on Signal and Information Processing, 3, 2014.

[5] L. Deng, G. Hinton, and B. Kingsbury. New types of deep neural network learning for speech recognition and related applications: an overview. In 2013 IEEE Inter-national Conference on Acoustics, Speech and Signal Processing, pages 8599\{8603, May 2013.

[6] S. Ghosh-Dastidar and H. Adeli. Spiking neural networks. International journal of neural systems, 19(04):295 \{308, 2009.

[7] N. Kalchbrenner, E. Grefenstette, and P. Blunsom. A convolutional neural network for modelling sentences. arXiv preprint arXiv:1404.2188, 2014

A. Krizhevsky, I. Sutskever, and G. E. Hinton. Imagenet classi cation with deep convolutional neural networks. In Advances in neural information processing sys-tems, pages 1097\{1105, 2012.

[8] Y. LeCun, L. Bottou, Y. Bengio, and P. Ha ner. Gradient-based learning applied to document recognition. Proceedings of the IEEE, 86(11):2278\{2324, 1998.

[9] W. Maass and C. M. Bishop. Pulsed neural networks. MIT press, 2001.
[10] Sutskever, O. Vinyals, and Q. V. Le. Sequence to sequence learning with neural networks. In Advances in neural information processing systems, pages 3104\{3112, 2014.

[11] Z. Wang, Y. Ma, F. Cheng, and L. Yang. Review of pulse-coupled neural networks. Image and Vision Computing, 28(1):5 \{13, 2010.

[12] R. J. Williams and D. Zipser. A learning algorithm for continually running fully recurrent neural networks. Neural computation, 1(2): $270\{280,1989$

[13] C. Zhang, P. Li, G. Sun, Y. Guan, B. Xiao, and J. Cong. Optimizing FPGA-based accelerator design for deep convolutional neural networks. In Proceedings of the 2015 ACM/SIGDA International Symposium on Field-Programmable Gate Arrays, pages 161\{170. ACM, 2015. 